\title{
HRAS Protein Variant
}

National Cancer Institute

\section{Source}

National Cancer Institute. HRAS Protein Variant. NCI Thesaurus. Code C140251.

A variation in the amino acid sequence for the GT Pase HRas protein. 